\title{
A Numerical Study on the Lid-Driven Cavity with Power-Law Fluids at Different Moving Lengths of the Top Lid
}

\author{
Prabir Sikdar ${ }^{1,}{ }^{*}$, Sunil Manohar Dash ${ }^{1}$ \\ 1 Department of Aerospace Engineering, Indian Institute of Technology Kharagpur, Kharagpur, India- 721 302, India
}

\section{ARTICLE INFO}

\section{Article history:}

Received 21 April 2020

Received in revised form 18 June 2020

Accepted 25 June 2020

Available online 30 June 2020

\section{Keywords:}

Power-law fluid; Lid-driven cavity;

\section{ABSTRACT}

In recent years, the lattice Boltzmann method has emerged as a promising numerical technique for solving complex fluid flow problems. This method has been successfully applied to both Newtonian and non-Newtonian fluids. Lately, the research in the field of the dynamics of non-Newtonian fluids inside a 2D square lid-driven cavity has attracted significant attention. In this numerical study, using the in-house developed Lattice Boltzmann solver, a two dimensional (2D) square lid-driven cavity with powerlaw fluids is investigated for different moving lengths of the top lid. Here, the top moving lid of length (L) is bisected into stationary (Lx) and moving (L-Lx) parts. The effects of the power-law index ( $n$ ), Reynolds number (Re) on the flow regime, velocity and vorticity distribution in the cavity are analyzed for $\mathrm{Lx}$ in the range $0.0 \mathrm{~L}$ to $0.9 \mathrm{~L}$. The flow regime in the cavity changes from Type-I (one primary vortex) to Type-II (two primary vortices) when the value of $L x$ is increased beyond a critical value. This critical value of $L x$ is found to depend on both $n$ and Re. We have further investigated the inertia effects of the moving lid and analyzed the trajectory of the primary vortex center as the functions of the power-law index ( $n$ ), Reynolds number (Re) and length of the moving lid.

\section{Introduction}

Since the non-Newtonian fluids have various industrial, engineering and biological applications, it is important to understand its fluid dynamics. Primarily, the non-linear relationship between the shear stress and strain rate makes the flow physics of the non-Newtonian fluid different from its Newtonian counterpart. To characterize the shear dependent flow behavior of the non-Newtonian fluids, several constitutive models have been proposed [1-3] out of which the Power-law model is widely used $[4,5]$.

In the present study, to investigate the flow dynamics, we have focused on a lid-driven cavity flow problem which has previously received significant attention in the CFD researcher community and also has various practical applications such as solar collector [6], short-dwell and flexible blade

\footnotetext{
* Corresponding author.

E-mail address: iamprabirsikdar@gmail.com (Prabir Sikdar)
} 
coaters [7], etc. The factors like Reynolds number (Re) [8-10], the number of the moving lids/sides [11] and orientation [12] of the cavity govern the flow regimes in the lid-driven cavity problem. Burggraf [13] analytically and numerically investigated the steady-state solution of the lid-driven square cavity flow for Reynolds number up to 400 and highlighted that the flow regime mainly contains a large primary vortex and two small corner vortices. In his study, the shifting of the vortex center from the top lid region to the geometric center was observed when Re is increased. To further investigate the cavity flow problem, Ghia et al., [8] have used the stream function-vorticity formulation of the NS equation at higher Re. Recently, the lattice Boltzmann method (LBM) with Bhatnagar-Gross-Krook (BGK) collision model has been used to solve the flow physics of the liddriven cavity problems $[9,11]$. Hou et al., [14] used the LBM with a single relaxation time (SRT) model and found that their results match quite well with conventional CFD results. Some of the later studies $[9,15]$ examined the lid-driven cavity flow with various aspect ratios and Reynolds number $(\mathrm{Re})$ with Newtonian fluid. They analyzed the influences of Re and aspect ratios of the cavity on the shape and size of the vortex, vortex center location, and the number of vortices in the cavity. In our recent study, [16] the effect of different moving lengths of the top lid of a square cavity is investigated for the Newtonian fluid. Distinct flow types are observed when the lid length is modified.

It is worth to note that the linear nature of the governing equations of LBM has several advantages over the conventional NS solver, such as ease in parallel code execution and implementation of the complex boundary conditions, etc. Therefore, LBM is not only followed in the case of Newtonian fluids but also widely adopted in solving non-Newtonian flow problems. Using LBM solvers, Gabbanelli et al., [17] have simulated the power-law fluid flow in the parallel plate geometry and captured the flow physics of shear-thinning and shear-thickening fluids. Boyd et al., [18] presented a new second-order accurate lattice Boltzmann method to simulate the shear dependent non-Newtonian flows through a rigid pipe and compared their results with the analytical solutions. Sulvian et al., [5] predicted the shear-thinning flow behavior of the power-law fluid in 2D and 3D porous media. They also discussed the selection of local lattice viscosity for a stable simulation. The power-law fluid flow inside the 2D square cavity using LBM-MRT (multi relaxation time) model is studied by Li et al., [19] for a wide range of power-law index (n) and Re. It is observed that the complicated nature of power-law fluid for different values of $n$ changed the flow structure, number of vortices and their strength inside the cavity. The simulation of power-law fluid for a double-sided lid-driven square cavity is studied by Mendu et al., [20]. The top and bottom walls have been moved and other walls of the cavity are kept fixed. They have studied the effect of $n$ and Re on the vortex center location and variation of velocity components in the cavity.

It is understood from the above literature review that the LBM can successfully be applied to simulate the flow of Newtonian as well as non-Newtonian fluid. Again, the flow physics of Newtonian fluid in the lid-driven square cavity has been well reported. To the best of the authors' knowledge, these studies are restricted to the cases where the length of the moving lid is identical to one side of the square cavity. However, it is important to understand the flow physics of the shear-thinning and shear-thickening fluid in the square lid-driven cavity for the various moving length lid lengths. This is very essential to characterize the flow mixing in the cavity. With this motivation, we have investigated the $2 \mathrm{D}$, top-lid-driven square cavity for various moving lid lengths using in-house developed LBM-SRT solver. Three different power-law indices are selected to capture the flow physics of both Newtonian and non-Newtonian fluids at low $(\mathrm{Re}=100)$, intermediate $(\mathrm{Re}=400)$ and high Reynolds number $(\mathrm{Re}=1000)$. 


\section{Numerical Methodology}

The governing equations for incompressible, steady, viscous, laminar and generalized nonNewtonian flow in the two-dimensional rectangular cavity is given by [19]

$\nabla_{\alpha} \cdot\left(\rho \boldsymbol{u}_{\alpha}\right)=0$

$\nabla_{\beta \alpha} \cdot\left(\boldsymbol{u}_{\alpha} \boldsymbol{u}_{\beta}\right)=-\nabla_{\alpha} P+\nabla_{\beta} \cdot \tau_{\alpha \beta}$

where the variables $\rho$ and $P$ denote the density and pressure of the fluid, respectively. The shear stress tensor is represented by $\tau_{\alpha \beta}$ and it is expressed as, $\tau_{\alpha \beta}=\mu(|\dot{\gamma}|) S_{\alpha \beta}$. Here, $\mu$ is called the apparent viscosity of the fluid. The variable $\dot{\gamma}$ and $S_{\alpha \beta}$ is called the shear rate and shear rate tensor, respectively. The expression for shear rate tensor is

$S_{\alpha \beta}=\frac{1}{2}\left(\nabla_{\alpha} u_{\beta}+\nabla_{\beta} u_{\alpha}\right)$

In this study, to relate the apparent viscosity with the shear rate, the power-law model is used.

\subsection{Power-Law Model}

The apparent viscosity $(\mu)$ in the power-law model for the non-Newtonian fluid is expressed as [18], $\mu(|\dot{\gamma}|)=m|\dot{\gamma}|^{n-1}$, where $m$ and $n$ are proportionality constant and power-law index, respectively. Here, the constant $m$ can be calculated from the expression of the Reynolds number i.e. $R e=\frac{U^{2-n} L^{n}}{m} \Rightarrow m=\frac{U^{2-n} L^{n}}{R e}$. Note that the variable $L$ and $U$ are the characteristics length and velocity scale, respectively. Furthermore, the shear rate $(\dot{\gamma})$ can be calculated using the relation [18], $\dot{\gamma}=$ $2 \sqrt{D_{\Pi}}$, where $D_{\Pi}$ is the second invariant of the strain rate tensor and is expressed as, $D_{\Pi}=$ $\sum_{\alpha, \beta=1}^{l} S_{\alpha \beta} S_{\alpha \beta}$. In the present 2D simulations the upper limit of $/$ is set as 2 . Eventually, the apparent viscosity simplifies to;

$\mu(|\dot{\gamma}|)=m|\dot{\gamma}|^{n-1}=\left(\frac{U^{2-n} L^{n}}{R e}\right)\left(2 \sqrt{D_{\Pi}}\right)^{n-1}$

\subsection{The Lattice Boltzmann Method}

In this study, the inhouse developed code based on the lattice Boltzmann method is followed to solve the above governing Eq. (1) and (2). The Lattice Boltzmann equation which is derived from the finite difference approximation of the Boltzmann equation is [18],

$f_{i}\left(\boldsymbol{r}+\boldsymbol{c}_{\boldsymbol{i}} \Delta t, t+\Delta t\right)-f_{i}(\boldsymbol{r}, t)=-\frac{f_{i}(\boldsymbol{r}, t)-f_{i}^{e q}(\boldsymbol{r}, t)}{\tau}$

For 2D simulations, we have used D2Q9 (2: two dimensions; 9: nine lattice directions) lattice model with BGK approximation $[18,20]$. Here, $f_{i}(\boldsymbol{r}, t)$ denotes the particle distribution function along $i$ direction at position $r$ and time $t$. $\tau$ is the single relaxation time factor. In LBM, the kinematic viscosity $(v)$ and relaxation time parameter $(\tau)$ are related as, $\tau=\frac{1}{2}+3 v \Rightarrow \tau=\frac{1}{2}+3\left\{\frac{\mu(|\dot{\gamma}|)}{\rho}\right\}$. Here, the value of $\tau$ is constant at every node within the domain for a Newtonian fluid. However, for power-law fluids, 
the apparent viscosity is dependent on the shear rate, which varies locally. Thus, both the kinematic viscosity $(v)$ as well as $\tau$ is a local variable. So, the above relationship becomes; $\tau(\boldsymbol{r}, t)=\frac{1}{2}+3 v(\boldsymbol{r}, t)$. Note that using the Chapman-Enskog expansion, the macroscopic governing Eq. (1) and (2) can be suitably recovered from the mesoscopic LBM Eq. (5). Additional details on LBM can be found in the ref. [18-21].

\subsection{Problem Definition and Boundary Condition}

The schematic diagram of a two-dimensional lid-driven cavity with the appropriate boundary conditions is shown in Figure 1. The top wall is divided into two parts in which one part is stationary and the other part is moving. The length of the stationary part is denoted by Lx. Here, $L x$ is varied from $0.1 \mathrm{~L}$ to $0.9 \mathrm{~L}$ with an interval of $0.1 \mathrm{~L}$. Three different power-law indices $(n=0.75, n=1.00, n=$ 1.25) are considered which covers the shear-thinning $(n<1)$, shear-thickening $(n>1)$, and Newtonian fluid $(n=1)$.

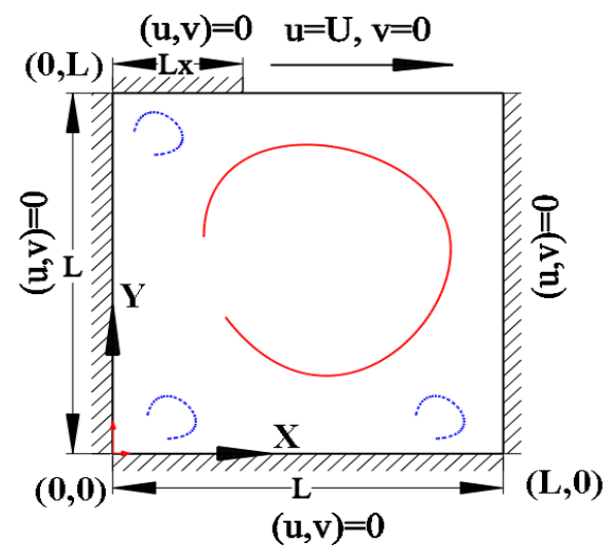

Fig. 1. Schematic diagram of the present lid-driven cavity flow problem

The application of boundary condition (BC) in LBM is different from the conventional NavierStokes solver in which the BC is directly applied at the boundary in terms of macroscopic variables. Whereas, in LBM, the particle density distribution function is used to apply BC. In this problem, for the stationary wall, the well-known bounce back technique is considered [20]. For the moving length of the top lid, the equilibrium distribution function is modified [14] to satisfy the moving wall condition.

\subsection{Numerical Execution}

At $t=0$, velocity components $(u, v)$ at all lattice nodes except on the top moving wall are taken as zero. The non-dimensional velocity of the moving part of the top wall is set as $u=0.1$. Here, the velocity of the moving lid is suitably selected so that the Mach number $(\mathrm{Ma}<0.2)$ of the flow lies in the incompressible limit. Also, the initial density at each grid point is set as 1.0 in the lattice unit. The convergence criteria (CC) shown in Eq. (6) for the velocity residue is set to $10^{-8}$.

$C C=\sum_{x, y} \frac{[\boldsymbol{u}(x, y, t+\Delta t)-\boldsymbol{u}(x, y, t)]}{\boldsymbol{u}(x, y, t+\Delta t)}$ 
where, $x=1, N x ; y=1, N y-1$. The number of grid points along $\mathrm{X}$ and $\mathrm{Y}$ direction is $N x$ and $N y$, respectively. The velocity at the geometric center of the cavity is plotted in Figure 2 for different values of $\mathrm{n}$. It shows that the solution approaches steady-state as the time (iteration) progresses and this is true for all the cases considered here.

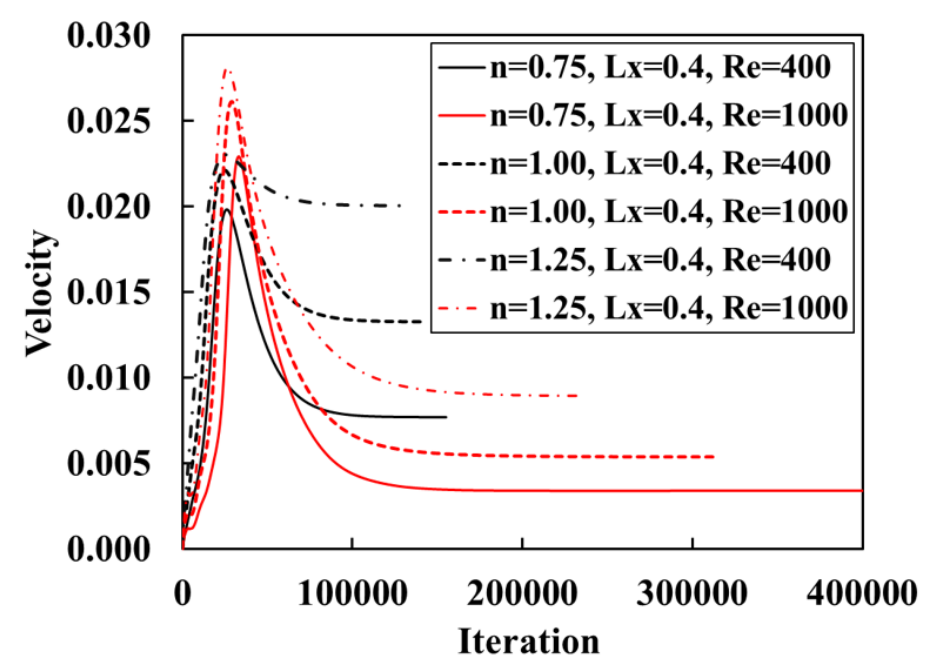

Fig. 2. The non-dimensional velocity vs. time (iteration) computed at the geometric center of the cavity for different values of $n, L x$, and Re

\subsection{Grid Independence Study and Validation of the LBM Code}

To ensure that the results of this study are independent of grid resolution, at first numerical simulations are performed on five different uniform grids of size $131 \times 131,201 \times 201,261 \times 261$, $331 \times 331$ and $391 \times 391$ on the square lid-driven cavity with $L x=0.4, n=0.75$ and $R e=400$. The vcomponent of velocity along the horizontal centreline of the cavity is compared in Figure 3 . It can be seen that the error is insignificant when the grid size is finer than $261 \times 261$. Hence, the grid size of $261 \times 261$ is chosen for the remaining simulation of $\operatorname{Re}=100$ and 400 . However, for higher resolution of the velocity and vorticity distributions, the grid size of $331 \times 331$ is selected for $\operatorname{Re}=1000$.

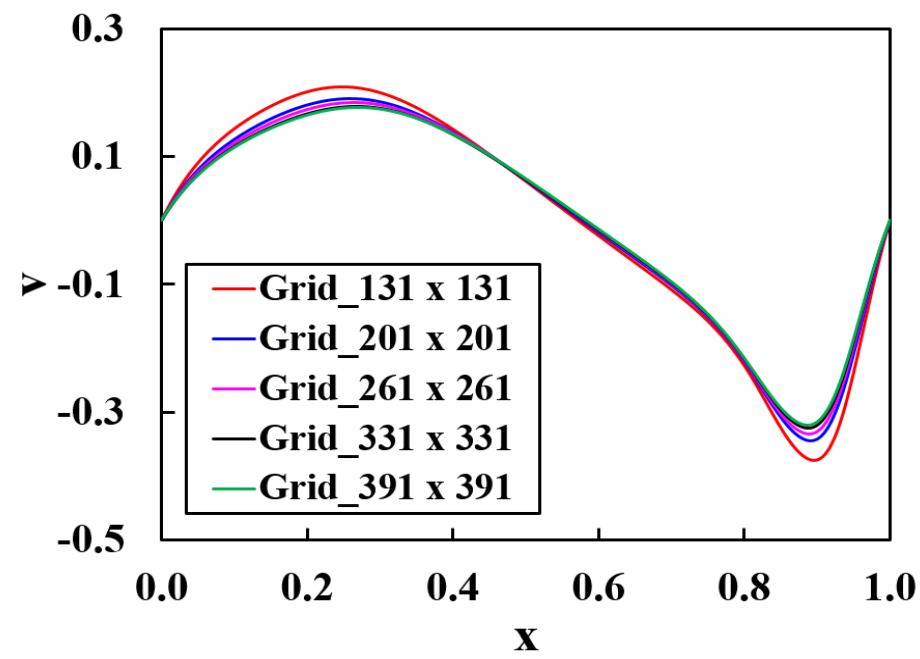

Fig. 3. Comparison of $v$ component velocity for different grid sizes along the horizontal centreline of the square cavity, for $L x=0.4, n=0.75$ and $R e=400$ 
To validate the present LBM solver, numerical simulations on the square lid-driven cavity are carried out for $\operatorname{Re}=100$ and at $L x=0.0 \mathrm{~L}$ with both the Newtonian and non-Newtonian fluids. For different cases, the computed $u$ and $v$ components of the velocity along the vertical and horizontal centreline of the cavity are compared with ref. $[8,19]$ in Figure 4 . It is observed that the velocity profiles obtained from the present solver match well with those published results which also confirms the accuracy of the developed solver.

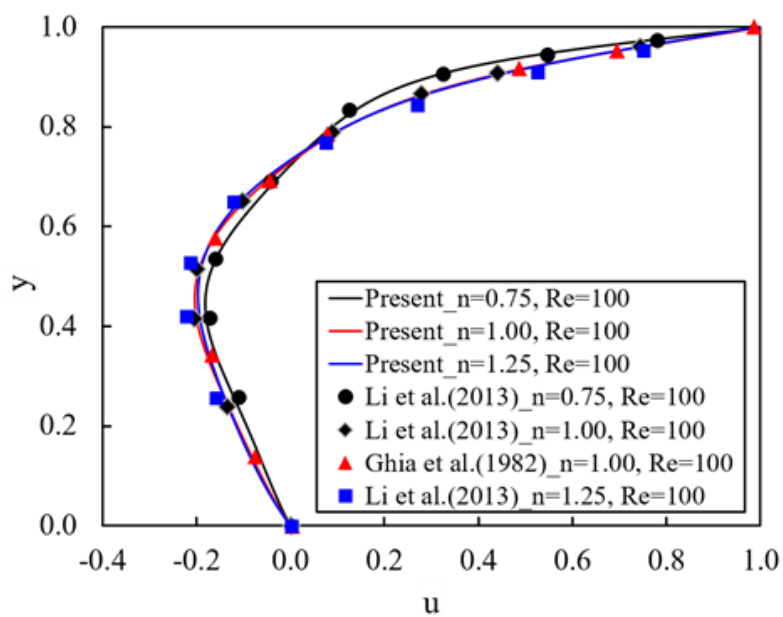

(a)

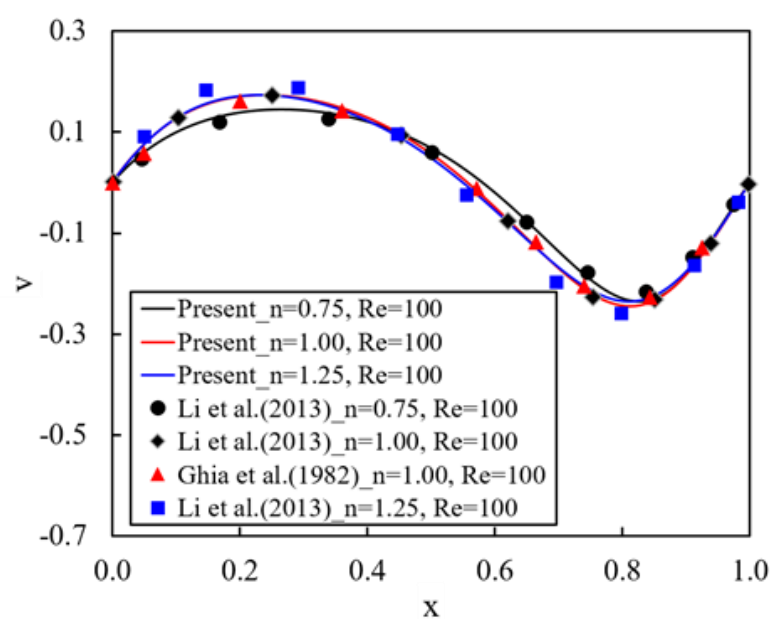

(b)

Fig. 4. Comparison of $u$ and $v$ components of the velocity along the vertical and horizontal centreline of the cavity

\section{Results and Discussion}

In the following section, the effects of the Reynolds number $(R e)$, Power-law index (n) of the fluid and the moving lid length (L-Lx) on the flow regime, velocity and vorticity distribution in the square cavity are investigated.

\subsection{Flow Regime}

In the case of both Newtonian and non-Newtonian fluids, the typical flow regime in the lid-driven square cavity consists of one large primary vortex and several small corner vortices $[15,19,20]$. In the present study, it is noticed that the flow regime in the cavity is modified when the moving lid length $(\mathrm{Lx}-\mathrm{L})$, Reynolds number (Re) and Power-law index $(\mathrm{n})$ of the non-Newtonian fluid is varied. We have broadly categorized the flow regime in the cavity as Type-I and Type-II based on the number of the primary vortex (PV).

As shown in the streamline plots in Figure 5, in Type-I and Type-Il flow regimes, one and two primary vortices are observed, respectively. Note that in both Type-I and Type-II flow regimes, several corner vortices are also seen. The red and blue color streamline indicates the clockwise and counterclockwise rotation of the vortices, respectively. In Figure 6, the observed flow types as functions of the Power-law index (n), moving lid length (L-Lx) and Reynolds number (Re) are shown. It is seen that at low $\mathrm{Re}$, the transition to Type-II flow regime appears for minimum moving lid length i.e. $\mathrm{Lx}=0.9 \mathrm{~L}$, at all $n$. However, at higher $\operatorname{Re}=1000$, the Type-II flow regime only appears for $n \geq 1$. 


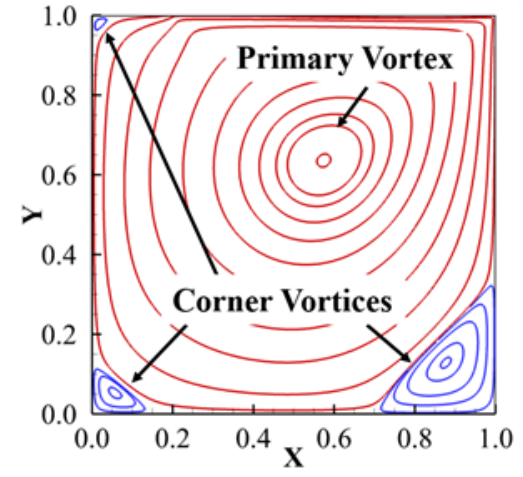

(a)

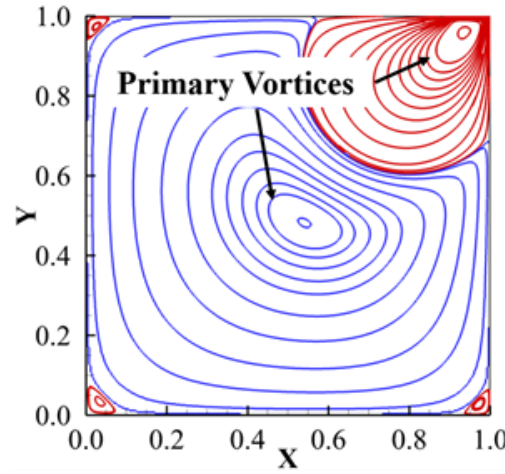

(b)

Fig. 5. Streamline patterns showing the (a) Type-I (for $L x=0.2, n=1.25$, $R e=400$ ) and (b) Type-II (for $L x=0.9, n=1.25, R e=400$ ) flow regimes, respectively. Here, the red and blue color streamline indicates the clockwise and counter-clockwise rotation of the vortices, respectively

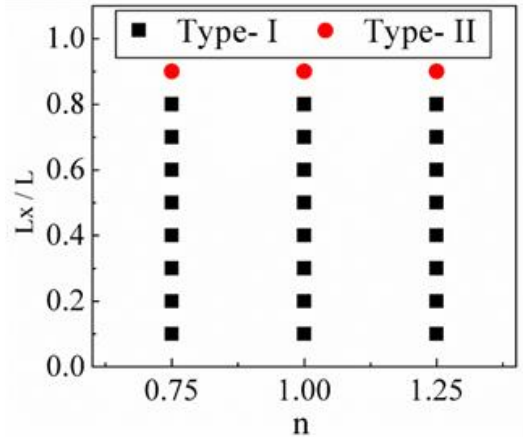

(a)

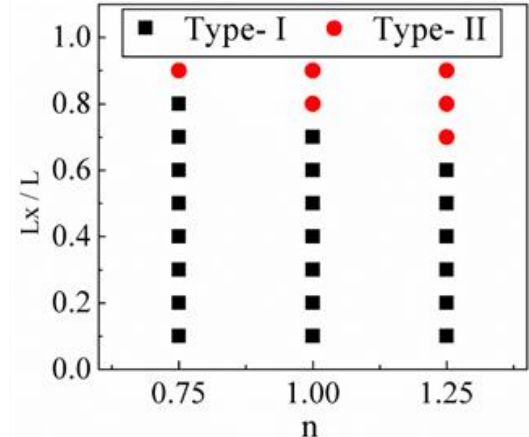

(b)

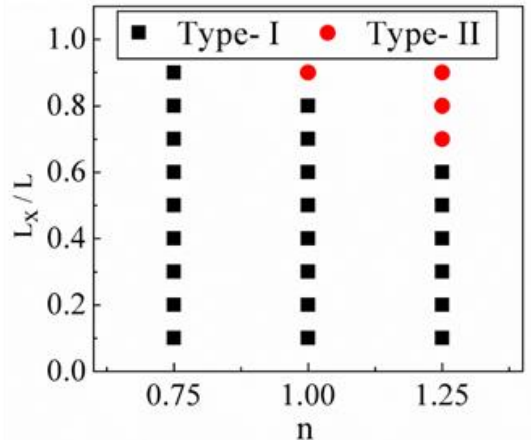

(c)

Fig. 6. Flow types observed at different $L x$ and $n$ for (a) $R e=100$, (b) $R e=400$ and (c) $\operatorname{Re}=1000$, respectively

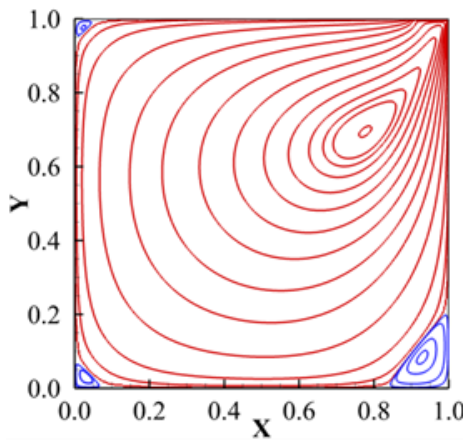

(a)

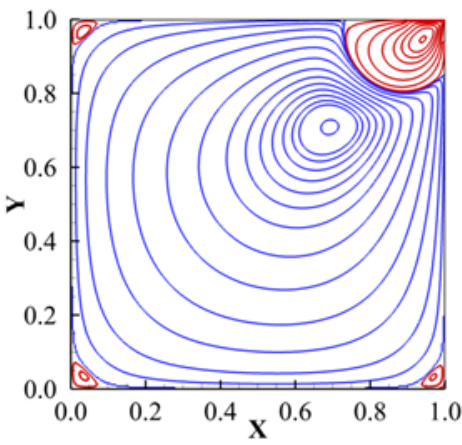

(b)

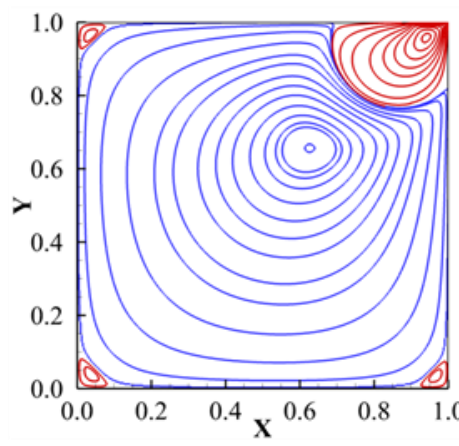

(c)

Fig. 7. Streamline patterns seen in the square lid-driven cavity at $R e=1000$ and $L x=0.9$ for (a) $n=0.75,(b) n=1.00$ and $(c) n=1.25$, respectively. Here, the red and blue color streamline indicates the clockwise and counter-clockwise rotation of the vortices, respectively

Moreover, for shear-thickening ( $n>1$ ) fluid, the transition to the Type-II flow regime occurs at intermediate moving lid length, i.e. $L x>0.6 \mathrm{~L}$. It is worth to note that the inertia effect of the moving lid primarily governs the flow regime transition. When the moving lid length is reduced and $\operatorname{Re}$ is increased, the inertia effects get concentrated towards the top-right corner (i.e. the location where the moving lid is defined, see in Figure 1 ) of the cavity. For the shear-thinning fluids $(n<1)$, the viscous effects decrease with the increase of shear rates. As a result, for $\operatorname{Re}=1000$, the inertia effects of the top moving lid propagates easily into the cavity and does not allow to form the second PV inside the cavity for $n=0.75$ and $L x=0.9$ (see in the streamline plots in Figure $7(a)$ ). 
Conversely, for the case of shear-thickening fluids $(n>1)$, the inertia effects of the top moving lid may not propagate easily in the vicinity of the cavity due to higher viscous effects at higher shear rates. Therefore, the two counter-rotating PVs are seen in the cavity for $L x>0.6 L$, regardless of the increase of Reynolds number (see in the streamline plots in Figure 7(c)).

\subsection{Trajectory of the Primary Vortex Centre}

It is noticed that in a particular flow type, the size and location of the primary and corner vortices are different when $L x, n$, and Re are varied. In Figure 8 , both the $X$ and $Y$ coordinates of the primary vortex (PV) center location is plotted as functions of the Power-law index (n), moving lid length (L-Lx) and Reynolds number. Here, the PV center is identified as the location in the cavity where both $u$ and $v$ velocity components are zero. It can be seen that for low Re and all values of $n$, the PV center gradually shift towards the top right corner of the cavity when the moving lid length is reduced (ref. Figure 8(a)). However, at higher Re, the shift in the PV center appears for the intermediate moving lid lengths, i.e. $L x>0.5 \mathrm{~L}$ (ref. Figure 8 (b) and (c)) and unlike the low Re case, the trajectory of the PV center differs for different values of $\mathrm{n}$. Note that in Figure 8 , the location of the second PV center is also plotted for the Type-Il flow regimes.

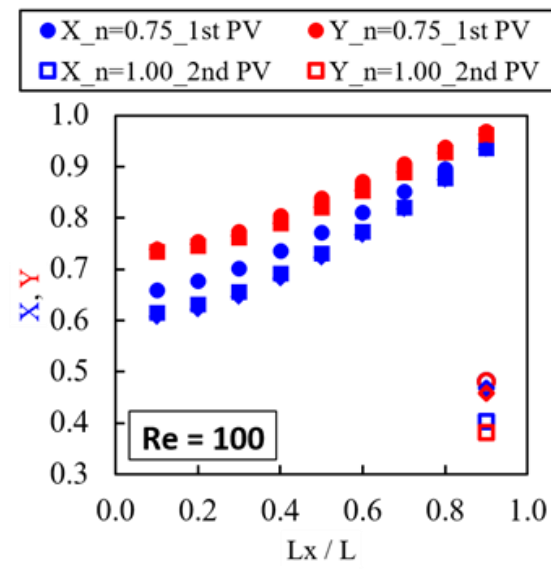

(a)

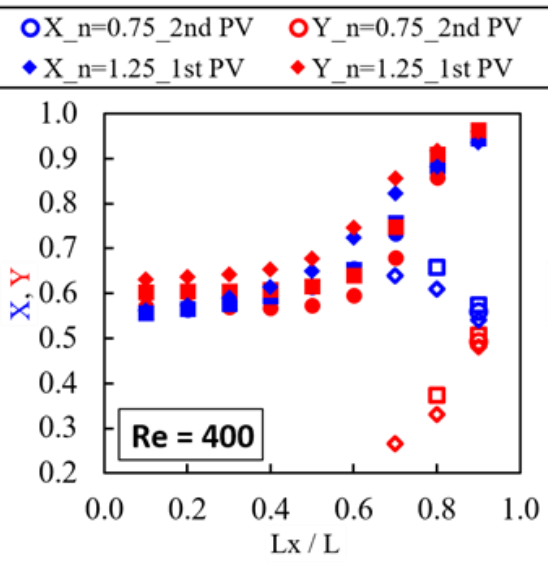

(b)

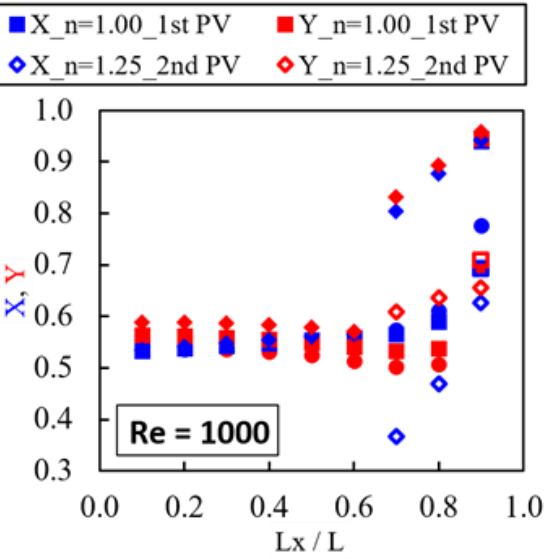

(c)

Fig. 8. Comparison of the primary vortex center location at different $L x$ and $n$, for (a) $\operatorname{Re}=100$, (b) $\operatorname{Re}=400$ and (c) $\operatorname{Re}=1000$, respectively

\subsection{Cavity Centerline Velocity Distribution}

In Figure 9 and 10, $u$ and $v$ component of the velocity along vertical and horizontal centreline of the cavity is plotted, respectively. For the Type-I flow, u component velocity near the top wall of the cavity is positive, whereas, near the bottom wall, it is negative (see in Figure 9(a)). Similarly, the $v$ component velocity near the left and right walls of the cavity is positive and negative, respectively (see in Figure 10(a)). This is because of the clockwise rotational sense (see in Figure 5(a)) of the primary vortex in the Type-I flow regime. 


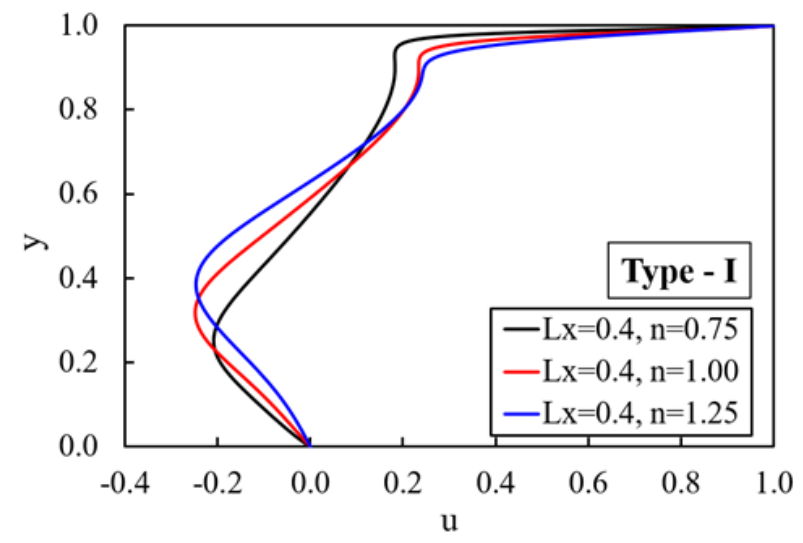

(a)

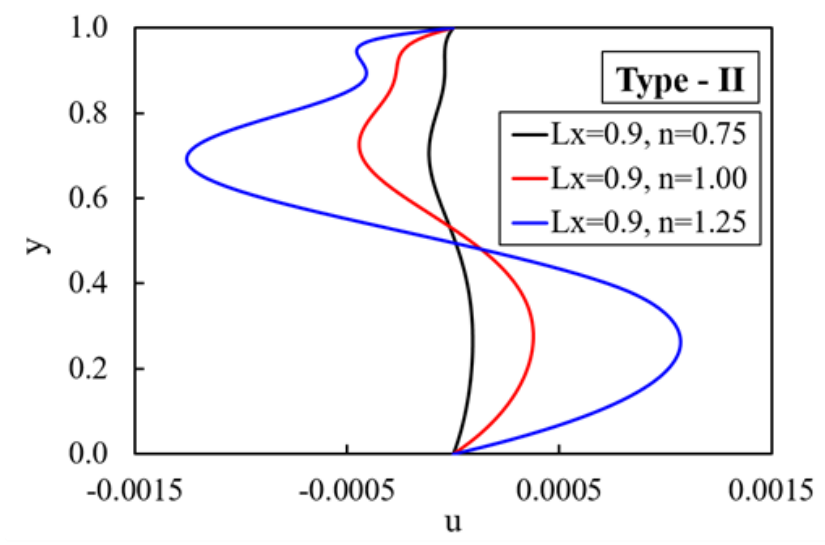

(b)

Fig. 9. The comparison of $u$-velocity along the vertical centreline of cavity at (a) $L x=0.4$ and (b) $L x=0.9$ for different values of $n$ and $\operatorname{Re}=400$

As shown in the streamline plot in Figure 5(b), in the case of Type-Il flow, the top and bottom PVs rotate in opposite directions. Since the bottom PV rotates in the counter-clockwise direction, $u$ component velocity near the bottom of the cavity is positive and becomes negative as it approaches the top lid (see in Figure 9(b)). Similarly, due to the interactions of the two counter-rotating vortices in the cavity, $v$ component velocity near the left wall is negative and it is positive close to the right wall (see in Figure 10(b)). It is worth to note that the trends of the velocity profiles are similar in the respective flow types for different values of $n$; however, their magnitude depends on the strength of vortices in the cavity.

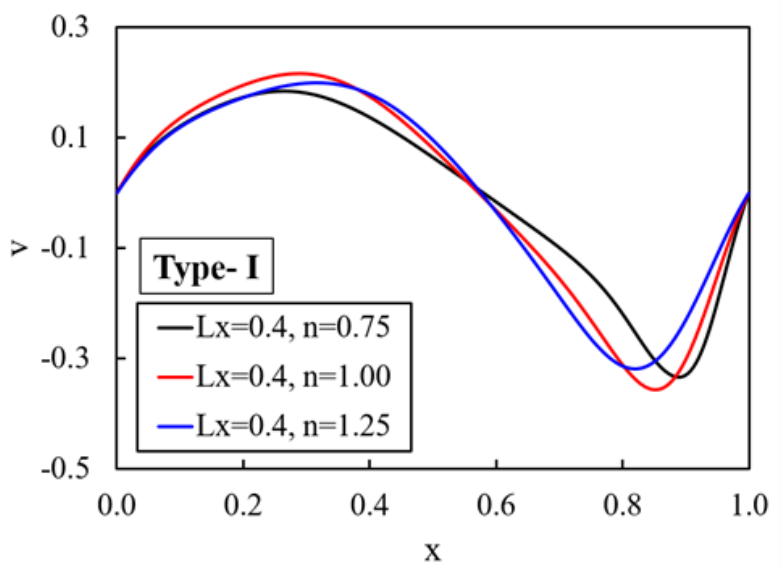

(a)

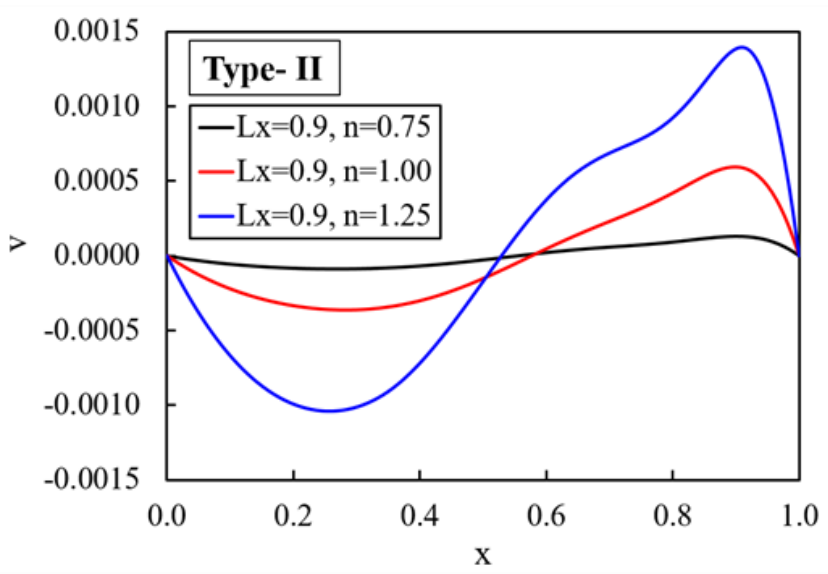

(b)

Fig. 10. The comparison of $v$-velocity along the horizontal centreline of cavity at (a) $L x=0.4$ and (b) $L x=$ 0.9 for different values of $n$ and $\operatorname{Re}=400$

\section{Conclusions}

In this study, using the power-law model and inhouse developed LBM solver, non-Newtonian and Newtonian fluid flows in a 2D square lid-driven cavity is investigated for the different moving lengths of the top lid and Reynolds number. The viscous effect changed by the power-law index and inertia effect changed by the moving lid length causes the shift in the primary and corner vortex centers in the cavity region and also modifies the flow regime from Type- I (one PV) to Type- II (two PVs). The velocity distribution along the centerline of the cavity shows a distinct pattern for Type-I and Type-II 
regime. The present study can be further extended to analyze the more complicated rheological behavior of other shear-thinning and shear-thickening fluid in a three-dimensional cavity model.

\section{Acknowledgment}

The first author is thankful to the Indian Institute of Technology Kharagpur (IIT Kharagpur) for providing computational resources of the present work. This research was not funded by any grant.

\section{References}

[1] Neofytou, Panagiotis. "A 3rd order upwind finite volume method for generalised Newtonian fluid flows." Advances in Engineering Software 36, no. 10 (2005): 664-680.

https://doi.org/10.1016/j.advengsoft.2005.03.011

[2] Boyd, Joshua, James M. Buick, and Simon Green. "Analysis of the Casson and Carreau-Yasuda non-Newtonian blood models in steady and oscillatory flows using the lattice Boltzmann method." Physics of Fluids 19, no. 9 (2007): 093103.

https://doi.org/10.1063/1.2772250

[3] Yoshino, Masato, Yoh-hei Hotta, Takafumi Hirozane, and Morinobu Endo. "A numerical method for incompressible non-Newtonian fluid flows based on the lattice Boltzmann method." Journal of Non-Newtonian Fluid Mechanics 147, no. 1-2 (2007): 69-78.

https://doi.org/10.1016/j.jnnfm.2007.07.007

[4] Chai, Zhenhua, Baochang Shi, Zhaoli Guo, and Fumei Rong. "Multiple-relaxation-time lattice Boltzmann model for generalized Newtonian fluid flows." Journal of Non-Newtonian Fluid Mechanics 166, no. 5-6 (2011): 332-342. https://doi.org/10.1016/i.jnnfm.2011.01.002

[5] Sullivan, S. P., L. F. Gladden, and M. L. Johns. "Simulation of power-law fluid flow through porous media using lattice Boltzmann techniques." Journal of Non-Newtonian Fluid Mechanics 133, no. 2-3 (2006): 91-98.

https://doi.org/10.1016/j.jnnfm.2005.11.003

[6] Zdanski, P. S. B., M. A. Ortega, and Nide GCR Fico Jr. "Numerical study of the flow over shallow cavities." Computers and Fluids 32, no. 7 (2003): 953-974.

https://doi.org/10.1016/S0045-7930(02)00067-1

[7] Aidun, Cyrus K., N. G. Triantafillopoulos, and J. D. Benson. "Global stability of a lid-driven cavity with throughflow: Flow visualization studies." Physics of Fluids A: Fluid Dynamics 3, no. 9 (1991): 2081-2091.

https://doi.org/10.1063/1.857891

[8] Ghia, U. K. N. G., Kirti N. Ghia, and C. T. Shin. "High-Re solutions for incompressible flow using the Navier-Stokes equations and a multigrid method." Journal of Computational Physics 48, no. 3 (1982): 387-411. https://doi.org/10.1016/0021-9991(82)90058-4

[9] Lin, Li-Song, Yi-Cheng Chen, and Chao-An Lin. "Multi relaxation time lattice Boltzmann simulations of deep lid driven cavity flows at different aspect ratios." Computers and Fluids 45, no. 1 (2011): 233-240.

https://doi.org/10.1016/j.compfluid.2010.12.012

[10] Jahanshaloo, L., NA Che Sidik, and S. Salimi. "Numerical simulation of high Reynolds number flow in lid-driven cavity using multi-relaxation time Lattice Boltzmann Method." Journal of Advanced Research in Fluid Mechanics and Thermal Sciences 24, no. 1 (2016): 12-21.

[11] Arun, S., and A. Satheesh. "Analysis of flow behaviour in a two sided lid driven cavity using lattice boltzmann technique." Alexandria Engineering Journal 54, no. 4 (2015): 795-806.

https://doi.org/10.1016/j.aej.2015.06.005

[12] Cheng, T. S., and W-H. Liu. "Effects of cavity inclination on mixed convection heat transfer in lid-driven cavity flows." Computers and Fluids 100 (2014): 108-122.

https://doi.org/10.1016/i.compfluid.2014.05.004

[13] Burggraf, Odus R. "Analytical and numerical studies of the structure of steady separated flows." Journal of Fluid Mechanics 24, no. 1 (1966): 113-151. https://doi.org/10.1017/S0022112066000545

[14] Hou, Shuling, Qisu Zou, Shiyi Chen, Gary Doolen, and Allen C. Cogley. "Simulation of cavity flow by the lattice Boltzmann method." Journal of Computational Physics 118, no. 2 (1995): 329-347. https://doi.org/10.1006/icph.1995.1103

[15] Patil, D. V., K. N. Lakshmisha, and B. Rogg. "Lattice Boltzmann simulation of lid-driven flow in deep cavities." Computers and Fluids 35, no. 10 (2006): 1116-1125.

https://doi.org/10.1016/j.compfluid.2005.06.006 
[16] Sikdar, Prabir, Sunil Manohar Dash, Kalyan Prasad Sinhamahapatra. "Lattice Boltzmann Simulations of a Lid-Driven Cavity at Different Moving Lengths of the Top Lid." In 46th National Conference on Fluid Mechanics and Fluid Power (FMFP), p, 32. 2019.

[17] Gabbanelli, Susana, German Drazer, and Joel Koplik. "Lattice Boltzmann method for non-Newtonian (power-law) fluids." Physical Review E 72, no. 4 (2005): 046312. https://doi.org/10.1103/PhysRevE.72.046312

[18] Boyd, J., James Buick, and Simon Green. "A second-order accurate lattice Boltzmann non-Newtonian flow model." Journal of Physics A: Mathematical and General 39, no. 46 (2006): 14241. https://doi.org/10.1088/0305-4470/39/46/001

[19] Li, Qiuxiang, Ning Hong, Baochang Shi, and Zhenhua Chai. "Simulation of power-law fluid flows in two-dimensional square cavity using multi-relaxation-time lattice Boltzmann method." Communications in Computational Physics 15, no. 1 (2014): 265-284. https://doi.org/10.4208/cicp.160212.210513a

[20] Mendu, Siva Subrahmanyam, and P. K. Das. "Flow of power-law fluids in a cavity driven by the motion of two facing lids-A simulation by lattice Boltzmann method." Journal of Non-Newtonian Fluid Mechanics 175 (2012): 10-24. https://doi.org/10.1016/j.jnnfm.2012.03.007

[21] Succi, Sauro. The lattice Boltzmann equation: for fluid dynamics and beyond. Oxford university press, 2001. https://doi.org/10.1016/S0997-7546(02)00005-5 